\title{
CT Urography for the Diagnosis of Medullary Sponge Kidney
}

\author{
Farrukh M. Koraishy ${ }^{a}$ Thuy-Trang T. Ngo ${ }^{c}$ Gary M. Israel ${ }^{b}$ Neera K. Dahl ${ }^{a}$ \\ ${ }^{a}$ Section of Nephrology and ${ }^{b}$ Diagnostic Radiology, Yale University School of Medicine, New Haven, Conn., and \\ 'Internal Medicine, Norwalk Hospital, Norwalk, Conn., USA
}

\section{Key Words}

Medullary sponge kidney $\cdot$ CT urography $\cdot$ Kidney stone

\begin{abstract}
Background: Medullary sponge kidney (MSK) is characterized by malformation of the terminal collecting ducts and is associated with an increased risk of nephrolithiasis, nephrocalcinosis, urinary tract infections, renal acidification defects, and reduced bone density. It has been historically diagnosed with intravenous pyelography (IVP), which is falling out of favor as an imaging modality. CT urography (CTU) performed with multidetector CT (MDCT) has been shown to create images of the renal collecting system with similar detail as IVP; however, its utility in diagnosing MSK has not been defined. Case Report: We present the first 15 patients with recurrent symptomatic nephrolithiasis who were evaluated in our renal stone clinic with CTU. Four patients were diagnosed with MSK after visualization of the characteristic radiologic findings. Discussion: CTU effectively demonstrates the characteristic radiologic findings of MSK including collecting tubule dilatation, medullary nephrocalcinosis, nephrolithiasis, and medullary cysts. Dose reduction protocols can reduce radiation exposure below that associated with conventional IVP. We propose CTU be considered for the diagnosis of MSK.
\end{abstract}

๑) 2014 S. Karger AG, Basel

\section{KARGER}

(C) 2014 S. Karger AG, Base

0250-8095/14/0392-0165\$39.50/0

E-Mail karger@karger.com

www.karger.com/ajn

\section{Background}

Medullary sponge kidney (MSK) is a congenital malformation characterized by cystic dilatation of the precalyceal collecting tubules in the renal pyramids that gives the medulla a sponge-like appearance on radiographic images [1] (summarized in table 1). MSK is classically known to be a benign condition that is incidentally found on renal imaging; however, since it has been strongly associated with various conditions that demand clinical attention, its diagnosis is important. MSK patients are known to have a high risk of renal nephrolithiasis [2], a condition that has been shown to increase the long-term risk of CKD [3]. MSK patients have also been reported to have reduced renal tubular function [4], a higher risk of urinary tract infections (UTIs), reduced bone density [5], and distal renal tubular acidosis (dRTA) [6]. While MSK is rare in children, it has been associated with growth failure [7].

The pathogenesis of MSK is not well defined. Although historically considered a sporadic condition, recent evidence shows a familial clustering of MSK patients in an autosomal dominant pattern [8]. This finding along with its association with congenital diseases [9] suggests a genetic etiology. The association of MSK with mutations in the developmental gene, glial cell-derived neurotrophic factor (GDNF), highlights a potential defect in nephron development [10].

The true prevalence of MSK is not known due to the nonspecific clinical features of the disease. While its over-

Farrukh M. Koraishy, MD

Yale University, School of Medicine, Section of Nephrology

BB114, 330 Cedar Street

New Haven, CT 06520 (USA)

E-Mail farrukh.koraishy@yale.edu 
Table 1. Summary of the key clinical features of MSK along with relevant references

\begin{tabular}{|c|c|c|}
\hline & Description & Reference \\
\hline Pathogenesis & $\begin{array}{l}\text { genetic } \\
\text { developmental defect }\end{array}$ & $\begin{array}{l}{[8]} \\
{[10]}\end{array}$ \\
\hline Prevalence & $\begin{array}{l}\text { general population }(0.5-1.0 \%) \\
\text { stone formers }(8.5 \%) \\
\text { calcium stone formers }(18.8 \% \text { women, } 11.7 \% \text { men })\end{array}$ & $\begin{array}{l}{[9]} \\
{[11]} \\
{[12]}\end{array}$ \\
\hline $\begin{array}{l}\text { Clinical features } \\
\text { and associated } \\
\text { conditions }\end{array}$ & $\begin{array}{l}\text { nephrolithiasis } \\
\text { nephrocalcinosis } \\
\text { reduced renal tubular function } \\
\text { UTIs } \\
\text { reduced bone density } \\
\text { dRTA (usually incomplete) } \\
\text { hematuria }\end{array}$ & $\begin{array}{l}{[11]} \\
{[12]} \\
{[4]} \\
{[9]} \\
{[5]} \\
{[6]} \\
{[15]}\end{array}$ \\
\hline Diagnosis & $\begin{array}{l}\text { IVP: highly sensitive for collecting tubule dilatation; risk of contrast } \\
\text { and radiation exposure and low sensitivity to detect small stones and } \\
\text { nephrocalcinosis; not routinely used anymore } \\
\text { NCCT: highly sensitive for stones, but low sensitivity to detect ectasia of } \\
\text { precalyceal tubules } \\
\text { Ultrasound: no exposure to contrast or radiation, but low sensitivity to } \\
\text { detect ectasia of precalyceal tubules } \\
\text { MRI: highly sensitive for medullary cysts, but expensive; not widely } \\
\text { available and insensitive for stones } \\
\text { CTU: highly sensitive for stones, medullary cysts, and collecting tubule } \\
\text { dilatation; risk of contrast and radiation exposure }\end{array}$ & $\begin{array}{l}{[9,20]} \\
{[10]} \\
{[21]} \\
{[23]}\end{array}$ \\
\hline Treatment & $\begin{array}{l}\text { nonspecific, manage risk factors } \\
\text { potassium citrate: for stone prevention and osteopenia }\end{array}$ & {$[5,13]$} \\
\hline
\end{tabular}

all prevalence in the general population is likely to be low (reported to be $0.5-1.0 \%$ ) [9], it is found quite frequently in patients with nephrolithiasis (8.5\%) [11], especially young women with calcium stones (18.8\%) [12].

Most cases of MSK are diagnosed during a workup of renal stones. However, a subset of MSK patients are clinically silent [2] and the diagnosis is incidentally picked up on renal imaging for other indications. The strong association of MSK with nephrolithiasis, especially calcium stones [12], is explained by the tendency of urinary stasis in the ectatic collecting ducts and the association of high quantities of stone promoters like hypercalciuria [13] and reduced levels of inhibitors like citrate and magnesium [14] in the urine. The presence of high urinary $\mathrm{pH}$ (due to dRTA) can specifically increase the risk for calcium phosphate precipitation and stone formation [6], and phosphate stones are seen more frequently than oxalate [13].

The risk of UTIs is thought to be due to the presence of urinary stasis and stones, and is particularly high in women [12]. The presence of struvite stones and obstruc- tive uropathy has been reported to be the most common cause of end-stage stage renal disease associated with MSK [3]. The hematuria can be microscopic or macroscopic and with or without the presence or stones [15]. Dysfunction of the renal collecting systems results in urinary acidification defects and decreased concentrating ability [6]. While overt acidosis and polyuria are rarely seen, incomplete dRTA and nocturia are common. Renal acidification has been proposed by Fabris et al. [9] to be the primary defect leading to the genesis of other metabolic abnormalities of MSK. The reason for reduced bone density in MSK is not clear [5]. While it has been postulated to be due to high urinary calcium loss, impaired urinary acidification, and hyperparathyroidism, the association of these findings with MSK has been inconsistent.

MSK treatment is nonspecific and related to the management of associated features. Metabolic abnormalities associated with MSK should be evaluated for and managed appropriately. Renal function and bone mineral density should be monitored and UTIs aggressively treat- 
ed. Potassium citrate has been shown to be effective in reducing stone risk [13] and improving bone density in MSK patients [5]. A potassium citrate dose of $10-20 \mathrm{ml} /$ day has been recommended to maintain 24-hour urinary levels $>450 \mathrm{mg} /$ day and a urine $\mathrm{pH}<7.5$ [9]. Most stones associated with MSK are small and pass spontaneously, but severe symptomatic stone disease might require invasive procedures like laser papillotomy [16].

\section{MSK Diagnosis}

MSK can be suspected on clinical grounds, but the diagnosis is radiographic [16]. While there is no established gold standard, it has been traditionally diagnosed with intravenous pyelography (IVP) performed in the setting of a renal colic. The high-resolution images obtained with IVP demonstrates pooling of contrast material within the dilated ectatic papillary ducts described as 'papillary brush' or a 'bouquet of flowers'. These radiographic findings along with the association of nephrolithiasis, nephrocalcinosis, and medullary cysts are used to make a diagnosis of MSK. Although the combination of these findings is highly suggestive for MSK, other etiologies of medullary cysts, hematuria, stones, and calcinosis should be considered and excluded.

Despite its extensive use for many years, the sensitivity and specificity of IVP for the diagnosis of MSK has been questionable. Some subtle cases can be missed due to suboptimal exam techniques, poor image quality resulting from bowel gas and stool, and low sensitivity to detect small stones [15]. On the other hand, the presence of homogeneous papillary 'blush' is of questionable significance [17], and it is not known whether it is an early manifestation of MSK or a normal variation.

The better sensitivity of noncontrast CT (NCCT) over IVP in the detection of kidney stones [18] and hematuria [15] has been well established. With the widespread use of the CT, IVP is rapidly falling out of favor as an imaging modality [19]. However, since papillary dilations in MSK are very thin, a high spatial resolution is required for their detection that can only be achieved with excretory urography. In a study comparing NCCT with IVP, Ginalski et al. [20] found that NCCT was not sensitive for detecting ectasia of precalyceal tubules, which is required for the diagnosis of MSK. On the other hand, they found that CT was more sensitive in detecting papillary calcifications.

Other imaging modalities have also not been found to be effective in diagnosing MSK. There are a few reported cases, especially in children, of the use of ultra- sound in MSK diagnosis [9], but the medullary hyperechogenicity and calcification seen is nonspecific. Recently, fat-saturated T2-weighted MRI [21] along with excretory urography was shown to allow MSK diagnosis; however, MRI is not as widely available and not in routine use in the workup of stone patients. Hence in centers where IVP is no longer performed, there is a strong concern that MSK might become a forgotten medical condition [22].

In 2007, Maw et al. [23] first reported that CT urography (CTU) can be used to diagnose MSK in a patient with kidney stones and UTIs. However, the use of CTU for MSK diagnosis has not been reported in a larger group of patients. We recently adopted CTU as the imaging modality of choice in our stone clinic for patients in whom we have a high suspicion of MSK. This is based on a history of recurrent symptomatic nephrolithiasis, especially if associated with one or more of the other clinical features of MSK including medullary calcinosis, hypercalciuria, hypocitraturia, or dRTA. We present 4 patients who were diagnosed with MSK out of the first 15 patients whom we referred for CTU.

\section{Case Report}

CTU was performed on a 16- or 64-slice MDCT scanner and the initial noncontrast images of the kidneys, ureters, and bladder were acquired and reconstructed at $3.75-\mathrm{mm}$ thickness. After $150 \mathrm{ml}$ of intravenous Omnipaque 300 (GE Medical Systems) followed by a $20-\mathrm{ml}$ normal saline flush, images of the kidneys were acquired during the nephrographic phase of enhancement (90-second scan delay) and reconstructed at $3.75-\mathrm{mm}$ thickness. Finally, excretory phase images were acquired after an 8 -min scan delay and reconstructed to $0.625-\mathrm{mm}$ thickness. Three-dimensional (3D) postprocessing was performed on a Vitrea workstation (Vital Images, Minnetonka, Minn, USA). The computed tomography dose index volume for patients ranged from 16.0 to $62.4 \mathrm{mGy}$.

The images were evaluated for medullary calcinosis, medullary cysts, and evidence of dilatation of the collecting tubules demonstrated by a papillary brush appearance or bouquet pattern. MSK was diagnosed solely on characteristic findings on the CTU images. The radiologist had no knowledge of the clinical history of the patients.

All patients had a history of recurrent symptomatic nephrolithiasis or medullary calcinosis (table 2). Patient 1 is a 51-year-old male with a history of incomplete dRTA and hypocitraturia who was found to have medullary nephrocalcinosis, medullary cysts, and a papillary brush pattern on CTU as shown in figure 1. Patient 2 is a 34-year-old female with a history of incomplete dRTA who had medullary nephrocalcinosis (fig. 2a) but no medullary cysts on the initial CTU images. However, the characteristic 'bouquet of flowers' pattern on the delayed excretory CTU images (fig. 2b) confirmed MSK. Patient 3 is a 53-year-old male with a history of incomplete dRTA and hypocitraturia who had medullary calcinosis and cysts in the initial CTU images and a papillary brush pattern 


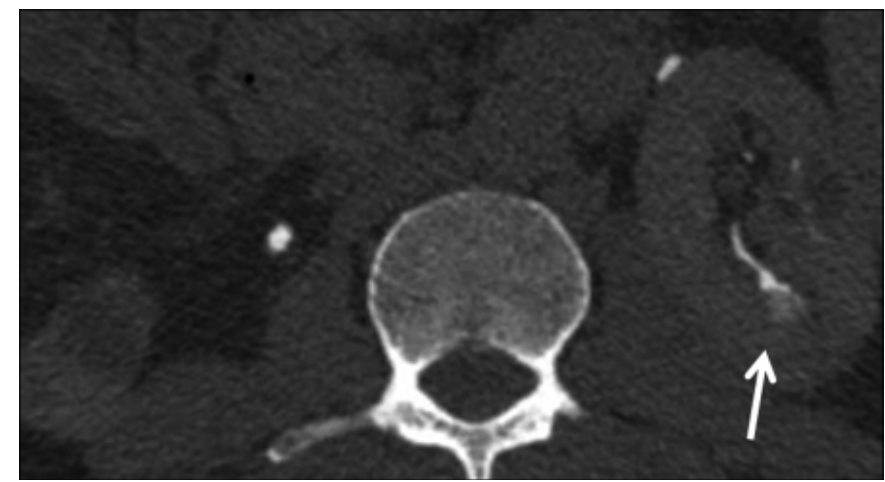

Fig. 1. Axial contrast-enhanced CT image during the excretory phase shows a papillary brush pattern in the medulla of the left kidney (arrow).

Table 2. A summary of the 15 patients with diagnostic features on CTU

\begin{tabular}{cllllll}
\hline Patient & Sex & Age & $\begin{array}{l}\text { Medullary } \\
\text { nephro- } \\
\text { calcinosis }\end{array}$ & $\begin{array}{l}\text { Medullary } \\
\text { cysts }\end{array}$ & $\begin{array}{l}\text { Papillary brush } \\
\text { or bouquet of } \\
\text { flowers pattern }\end{array}$ & MSK \\
\hline 1 & M & 51 & yes & yes & yes & yes \\
2 & F & 38 & yes & no & yes & yes \\
3 & M & 53 & yes & yes & yes & yes \\
4 & F & 37 & yes & yes & yes & yes \\
5 & M & 45 & yes & yes & no & no \\
6 & F & 46 & yes & no & no & no \\
7 & F & 46 & yes & no & no & no \\
8 & F & 28 & yes & no & no & no \\
9 & F & 53 & yes & no & no & no \\
10 & F & 27 & no & no & no & no \\
11 & F & 20 & no & no & no & no \\
12 & M & 36 & no & no & no & no \\
13 & F & 30 & no & no & no & no \\
14 & F & 47 & no & no & no & no \\
15 & F & 52 & no & no & no & no \\
\hline
\end{tabular}

All patents had a history of recurrent, symptomatic nephrolithiasis.

in the excretory images (fig. 3). Patient 4 is a 37 -year-old female with a history of symptomatic recurrent nephrolithiasis with UTIs, who was found to have medullary cysts and dilated collecting ducts creating a papillary brush pattern on CTU (fig. 4).

Of the remaining 11 patients who did not have the characteristic findings of collecting ductal dilatation to diagnose MSK, only 1 patient had medullary cysts. In summary medullary nephrocalcinosis was found in all, while medullary cysts were found in 3 of the 4 patients who were diagnosed with MSK based on CTU findings. The 4 patients diagnosed with MSK are being monitored closely for renal function, UTIs, bone disease, recurrence of symptomatic stones.
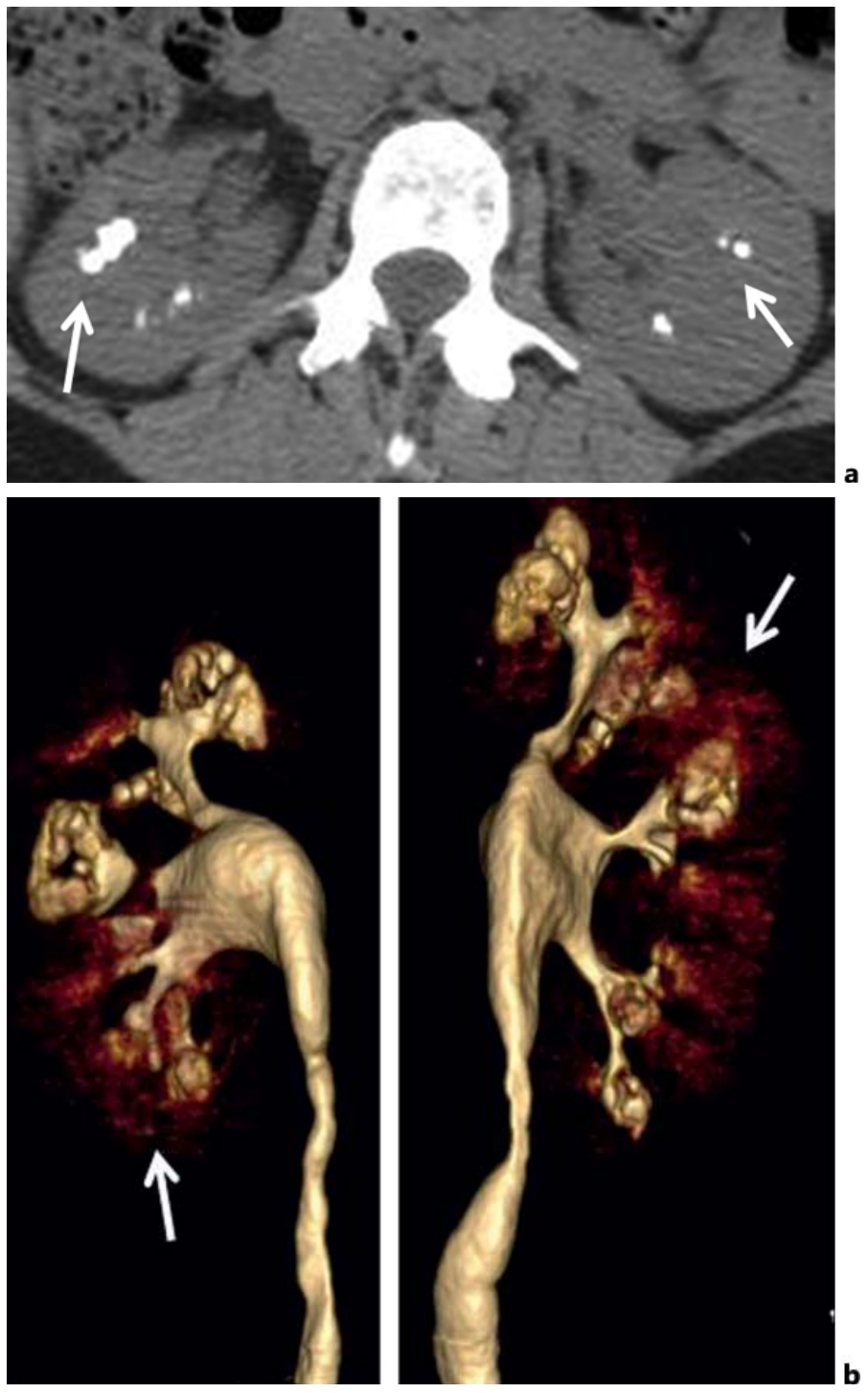

Fig. 2. a Noncontrast axial image at the level of the kidneys demonstrates medullary calcinosis (arrows) in both kidneys. b 3D volume rendered images created from CTU shows the classic 'bouquet of flowers' pattern (arrows) in the region of the papilla in both kidneys.

\section{Discussion}

In this report we present 4 patients with recurrent symptomatic kidney stones in whom we were able to diagnose MSK using CTU. We demonstrate that CTU with MDCT is able to delineate the collecting tubule dilatations and provide images of the characteristic 'brush' and 'bouquet of flowers' patterns with similar quality as reported with IVP. Within the same CTU study we were also able to obtain valuable information about the sever- 
ity of the stone disease, the extent of nephrocalcinosis, and the presence of medullary cysts that would not have been accurately obtained with IVP. These additional findings not only aid in making the diagnosis of MSK (to select patients for closer follow-up), but also provide information about any other significant abnormality in the genitourinary tract that could predispose these patients to future complications.

In most nephrolithiasis cases, it would probably not be appropriate to pursue a diagnosis of MSK with imaging due to the absence of any specific treatment and the risk of radiation exposure and contrast nephropathy associated with excretory urography. However, in selected cases of severe recurrent stone disease with other suggestive findings (as our patient cohort), it is useful to establish MSK to select patients for closer observation and treatment of risk factors for stones (e.g. hypocitraturia and hypercalciuria), UTIs, and osteopenia. Since tubular calcium disposition, recurrent infections, and obstructive uropathy can potentially lead to $\mathrm{CKD}$, renal function should also be monitored. Moreover, it has been shown that radiographic findings also have prognostic importance. In a retrospective study, Forster et al. [24] showed a strong correlation of the severity of radiologic and clinical features in MSK.

CTU utilizes MDCT technology along with the administration of intravenous contrast to create images similar those obtained with IVP. Due to this superior imaging capability, the European Society of Urogenital Radiology recommends CTU as the imaging modality of choice in the workup of hematuria, especially when urogenital cancer is suspected [25]. As we show in our images, this technique can also be highly effective in the detection of the various radiologic manifestations of MSK. The initial unenhanced images allow a detection of nephrocalcinosis and small stones (fig. 2a). The subsequent contrast-enhanced nephrographic phase gives high-resolution images of the kidneys that can detect medullary cysts (fig. 4). Excretory images combined with 3D reconstruction can demonstrate the characteristic findings of dilatation of the collecting tubules in MSK (fig. 1, 2b, 3, 4). However, due to multiple image acquisitions required, a major concern with CTU is the radiation exposure [26].

Radiation exposure associated with CTU should be minimized and new techniques are being used to achieve this goal. These include reduction in the tube current and voltage potential. In a study of 50 patients, Spielmann et al. [27] found that low-dose CT (both single- and multidetector) was comparable to standard dose in the detection of kidney and lower urinary tract stones, obstruction,

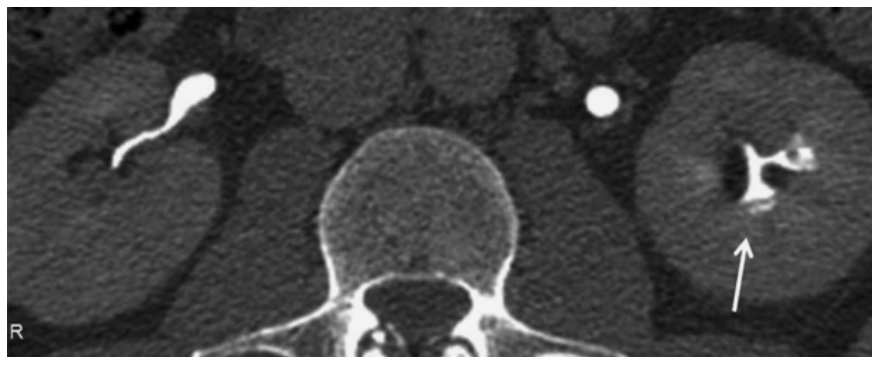

Fig. 3. Coronal reformation during the excretory phase showing a papillary brush pattern in the left renal medullary region (arrow).

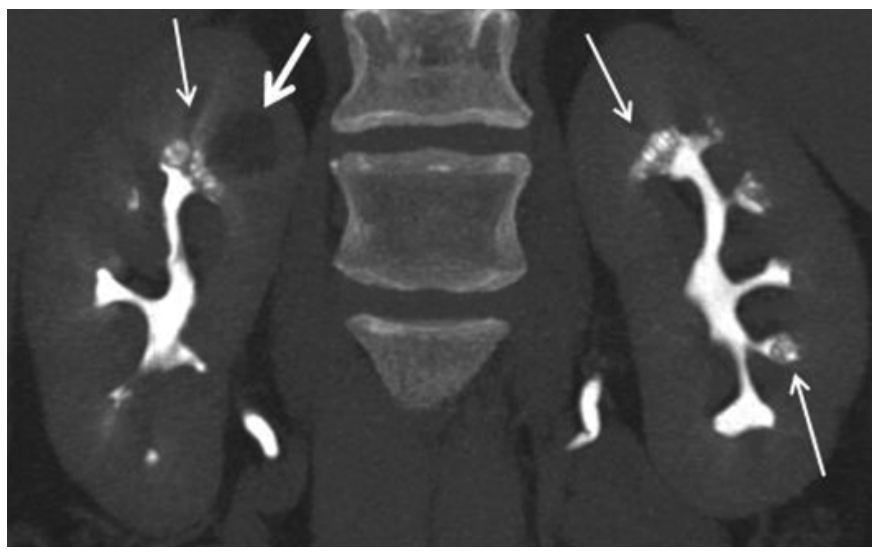

Fig. 4. Coronal maximum intensity projection during the excretory phase showing medullary cyst (thick arrow) and dilated collecting tubules showing a papillary brush pattern in both kidneys (thin arrows).

and other findings. In a recent study of 30 patients, lowdose CTU using the adaptive iterative dose reduction 3D technique resulted in $45 \%$ lower radiation exposure without any significant compromise in the image quality of the urinary tract [28]. Dual-energy split-bolus CT [29] and low voltage $(100 \mathrm{kVp})$ [30] are other evolving techniques used to reduce the radiation dose. Dahlman et al. [31] found that a combination of normal-dose corticomedullary phase with low-dose unenhanced and excretory phases resulted in a $42 \%$ reduction in radiation without image compromise. In our center, dose-saving techniques used for CTU include automatic tube current modulation and iterative reconstruction algorithms which are employed in all cases and reduce the radiation dose to levels lower than those associated with conventional IVP [26].

CTU is likely to become an accepted methodology for diagnosing MSK, particularly in centers which no longer routinely perform IVP. Our cases provide evidence in support of this approach. Moreover, the additional infor- 
mation provided by CTU (compared to IVP) might reduce the need for further imaging and hence radiation exposure to the patient.

As none of the 4 patients in this series received comparative IVP, we cannot make a definite conclusion about which modality is superior for MSK diagnosis. This question can only be answered in a blinded comparison trial of both imaging modalities in patients with recurrent symptomatic kidney stones. However, such a trial is unlikely to happen due to the additional radiation exposure that a second exam would entail.
In summary, this is the first reported series of patients with recurrent symptomatic nephrolithiasis and medullary calcinosis in which CTU has been shown to be effective in diagnosing MSK. We propose that CTU be considered the first-line test for the diagnosis of MSK in patients with a high index of suspicion.

\section{Disclosure Statement}

The authors have no potential conflicts of interest.

\section{References}

1 Lindvall N: Roentgenologic diagnosis of medullary sponge kidney. Acta Radiol 1959;51: 193-206.

2 McPhail EF, Gettman MT, Patterson DE, Rangel LJ, Krambeck AE: Nephrolithiasis in medullary sponge kidney: evaluation of clini$\mathrm{cal}$ and metabolic features. Urology 2012;79: 277-281.

3 Gambaro G, Favaro S, D’Angelo A: Risk for renal failure in nephrolithiasis. Am J Kidney Dis 2001;37:233-243.

4 Feest TG: Medullary sponge kidney: abnormalities of renal tubular and glomerular function, and their relationship to clinical features. Proc Eur Dial Transplant Assoc 1977; 14:511-517.

-5 Fabris A, Bernich P, Abaterusso C, Marchionna N, Canciani C, Nouvenne A, Zamboni M, Lupo A, Gambaro G: Bone disease in medullary sponge kidney and effect of potassium citrate treatment. Clin J Am Soc Nephrol 2009; 4:1974-1979.

6 Higashihara E, Nutahara K, Tago K, Ueno A, Niijima T: Medullary sponge kidney and renal acidification defect. Kidney Int 1984;25: 453-459.

7 Sluysmans T, Vanoverschelde JP, Malvaux P: Growth failure associated with medullary sponge kidney, due to incomplete renal tubular acidosis type 1. Eur J Pediatr 1987;146:78-80.

8 Fabris A, Lupo A, Ferraro PM, Anglani F, Pei Y, Danza FM, Gambaro G: Familial clustering of medullary sponge kidney is autosomal dominant with reduced penetrance and variable expressivity. Kidney Int 2013;83:272-277.

$\checkmark 9$ Fabris A, Anglani F, Lupo A, Gambaro G: Medullary sponge kidney: state of the art. Nephrol Dial Transplant 2013;28:1111-1119.

10 Torregrossa R, Anglani F, Fabris A, Gozzini A, Tanini A, Del Prete D, Cristofaro R, Artifoni L, Abaterusso C, Marchionna N, Lupo A, D'Angelo A, Gambaro G: Identification of GDNF gene sequence variations in patients with medullary sponge kidney disease. Clin J Am Soc Nephrol 2010;5:1205-1210.

-11 Laube M, Hess B, Terrier F, Vock P, Jaeger P: Prevalence of medullary sponge kidney in patients with and without nephrolithiasis (in
German). Praxis (Bern 1994) 1995;84:12241230.

12 Parks JH, Coe FL, Strauss AL: Calcium neph rolithiasis and medullary sponge kidney in women. N Engl J Med 1982;306:1088-1091.

13 Fabris A, Lupo A, Bernich P, Abaterusso C, Marchionna N, Nouvenne A, Gambaro G: Long-term treatment with potassium citrate and renal stones in medullary sponge kidney. Clin J Am Soc Nephrol 2010;5:1663-1668.

14 Yagisawa T, Kobayashi C, Hayashi T, Yoshida A, Toma H: Contributory metabolic factors in the development of nephrolithiasis in patients with medullary sponge kidney. Am J Kidney Dis 2001;37:1140-1143.

15 Lang EK, Macchia RJ, Thomas R, Watson RA, Marberger M, Lechner G, Gayle B, Richter F: Improved detection of renal pathologic features on multiphasic helical CT compared with IVU in patients presenting with microscopic hematuria. Urology 2003;61:528-532.

16 Gambaro G, Danza FM, Fabris A: Medullary sponge kidney. Curr Opin Nephrol Hypertens 2013;22:421-426.

17 Ginalski JM, Spiegel T, Jaeger P: Use of lowosmolality contrast medium does not increase prevalence of medullary sponge kidney. Radiology 1992;182:311-314.

18 Sourtzis S, Thibeau JF, Damry N, Raslan A, Vandendris M, Bellemans M: Radiologic investigation of renal colic: unenhanced helical CT compared with excretory urography. AJR Am J Roentgenol 1999;172:1491-1494.

19 Franco A, Tomas M, Alonso-Burgos A: Intravenous urography is died. Long live the computerized tomography (in Spanish)! Actas Urol Esp 2010;34:764-774.

20 Ginalski JM, Schnyder P, Portmann L, Jaeger P: Medullary sponge kidney on axial computed tomography: comparison with excretory urography. Eur J Radiol 1991;12:104-107.

21 Hida T, Nishie A, Asayama Y, Ishigami K, Fujita N, Inokuchi J, Naito S, Ando S, Honda H: MR imaging of focal medullary sponge kidney: case report. Magn Res Med Sci 2012;11: 65-69.

22 Stratta P, Fenoglio R, Quaglia M, Lazzarich E, Airoldi A: The missing medullary sponge kid- ney. Kidney Int 2009;76:459-460, author reply 460 .

23 Maw AM, Megibow AJ, Grasso M, Goldfarb DS: Diagnosis of medullary sponge kidney by computed tomographic urography. Am J Kidney Dis 2007;50:146-150.

24 Forster JA, Taylor J, Browning AJ, Biyani CS: A review of the natural progression of medullary sponge kidney and a novel grading system based on intravenous urography findings. Urol Int 2007;78:264-269.

25 Van Der Molen AJ, Cowan NC, Mueller-Lisse UG, Nolte-Ernsting CC, Takahashi S, Cohan $\mathrm{RH}$; CT Urography Working Group of the European Society of Urogenital Radiology (ESUR): CT urography: definition, indications and techniques. A guideline for clinical practice. Eur Radiol 2008;18:4-17.

26 Nawfel RD, Judy PF, Schleipman AR, Silverman SG: Patient radiation dose at CT urography and conventional urography. Radiology 2004;232:126-132.

27 Spielmann AL, Heneghan JP, Lee LJ, Yoshizumi T, Nelson RC: Decreasing the radiation dose for renal stone CT: a feasibility study of single- and multidetector CT. AJR Am J Roentgenol 2002;178:1058-1062.

28 Juri H, Matsuki M, Itou Y, Inada Y, Nakai G, Azuma H, Narumi Y: Initial experience with adaptive iterative dose reduction $3 \mathrm{D}$ to reduce radiation dose in computed tomographic urography. J Comput Assist Tomogr 2013;37: 52-57.

29 Takeuchi M, Kawai T, Ito M, Ogawa M, Ohashi K, Hara M, Shibamoto Y: Split-bolus CT-urography using dual-energy CT: feasibility, image quality and dose reduction. Eur J Radiol 2012;81:3160-3165.

30 Lee S, Jung SE, Rha SE, Byun JY: Reducing radiation in CT urography for hematuria: effect of using 100 kilovoltage protocol. Eur J Radiol 2012;81:e830-e834.

31 Dahlman P, van der Molen AJ, Magnusson M, Magnusson A: How much dose can be saved in three-phase CT urography? A combination of normal-dose corticomedullary phase with low-dose unenhanced and excretory phases. AJR Am J Roentgenol 2012;199:852-860. 